\title{
BMJ Open Opioid maintenance treatment in the Czech Republic, Norway and Denmark: a study protocol of a comparative registry linkage study
}

\author{
Roman Gabrhelík (D) , , Marte Handal, ${ }^{1,3}$ Viktor Mravčík, ${ }^{1}$ Blanka Nechanská, ${ }^{1,2}$ \\ Christian Tjagvad, ${ }^{4}$ Birgitte Thylstrup, ${ }^{5}$ Morten Hesse, ${ }^{5}$ Jakub Minař́k, ${ }^{1}$ \\ Jiří Jarkovský, ${ }^{1}$ Anne Bukten, ${ }^{4}$ Thomas Clausen, ${ }^{4}$ Svetlana Skurtveit ${ }^{3,4}$
}

To cite: Gabrhelík R, Handal M, Mravčík V, et al. Opioid maintenance treatment in the Czech Republic, Norway and Denmark: a study protocol of a comparative registry linkage study. BMJ Open 2021;11:e047028. doi:10.1136/ bmjopen-2020-047028

- Prepublication history for this paper is available online. To view these files, please visit the journal online (http://dx.doi org/10.1136/bmjopen-2020047028).

Received 18 November 2020 Revised 05 April 2021 Accepted 16 April 2021

Check for updates

(C) Author(s) (or their employer(s)) 2021. Re-use permitted under CC BY-NC. No commercial re-use. See rights and permissions. Published by BMJ.

${ }^{1}$ Department of Addictology, First Faculty of Medicine, Charles University, Prague, Czech Republic

${ }^{2}$ Department of Addictology, General University Hospital in Prague, Prague, Czech Republic ${ }^{3}$ Norwegian Institute of Public Health, Oslo, Norway

${ }^{4}$ Norwegian Centre for Addiction Research, University of OsIo, Oslo, Norway

${ }^{5}$ Centre For Alcohol And Drug Research, Aarhus University, Aarhus, Denmark

Correspondence to Professor Roman Gabrhelík; roman.gabrhelik@|f1.cuni.cz

\section{ABSTRACT}

Introduction Opioid maintenance treatment (OMT) varies across settings and between countries. We plan to use data from several nationwide health and population registers to further improve the knowledge base established from earlier studies. Our aim is to study OMT adherence trajectories and to identify factors associated with improved outcomes for OMT patients across the Czech Republic, Norway and Denmark, in order to further improve OMT and our understanding of the key elements of treatment success.

Methods and analysis The registry-based cohort approach across the three countries allows us to link data from a range of registers on the individual level, by using personal identifiers in nationwide cohorts of OMT and non-OMT patients and the general non-using populations. A total of $\sim 21500$ OMT patients over the last two decades in all three countries will be included in the study. The following outcome variables (based on the International Classification of Diseases, 10th Revision codes) will be obtained from relevant registers: treatment adherence to OMT, comorbidity (somatic and mental health), and all-cause and cause-specific mortality. Outcomes of the country-specific analyses will be pooled.

Ethics and dissemination The national OMT cohorts have been approved by the ethics committees in the respective countries. Data will be stored according to national and local guidelines and treated confidentially, and all data will be analysed separately for each country and compared across countries. Findings will be disseminated in peer-reviewed scientific journals, national and international conferences, and in briefings to inform clinical decision-making.

\section{INTRODUCTION}

Globally, opioid use disorders (OUDs) are a major public health concern, associated with high costs to the society, the healthcare system, and at the individual level for both the opioid users and their close ones. ${ }^{1-3}$ OUD is associated with high somatic comorbidity, including infectious diseases, such as HIV and hepatitis, ${ }^{34}$ high rates of psychiatric
Strengths and limitations of this study

- International registry linkage-based study design minimises selection and recall bias and makes it possible to follow individuals over time in treatment and out of treatment without loss to follow-up.

- The wide range of nationwide data from health and population-based registers in the Czech Republic, Norway and Denmark increases the generalisability of common findings.

- The possibility of performing meta-analysis of the results from multiple countries increases statistical power and makes it possible to study differences between the different opioid maintenance treatment (OMT) drugs (methadone, buprenorphine and buprenorphine with naloxone).

- The main limitation is that important information can be under-reported, reported in insufficient format or completely lacking in the national health registers.

- In Norway, OMT is defined by dispensed OMT drugs as recorded in the prescription database, while both the Czech Republic and Denmark have specific detailed registers for OMT treatment.

comorbidities, ${ }^{56}$ criminal activities, ${ }^{78}$ social exclusion and low quality of life of opioid users and significant others, economic costs, and loss of social cohesion. ${ }^{9-11}$ Furthermore, the rate of drug-related mortality is high $^{3}{ }^{12}$ including overdose deaths among young people with OUD. ${ }^{13}$

OUD requires long-term treatment that typically consists of opioid maintenance treatment (OMT) as the method of choice, including OMT medications in combination with psychosocial care. ${ }^{14}$ Methadone and buprenorphine are currently the most widely used opioids in OMT in Europe and elsewhere. ${ }^{15}$ Recent reports show that in Europe, methadone is received by $61 \%$, buprenorphine by $37 \%$ and other types of medication by $2 \%$ of patients in OMT. ${ }^{16}$ OMT 
is a well-established treatment approach for OUD, and has proven positive effects on a number of outcomes in various subpopulations, ${ }^{17} 18$ including positive effects on stabilisation of people with OUD, reducing the risk of relapse to illicit opioid use ${ }^{19}$ health-related variables such as (co)morbidity and mortality, ${ }^{20-25}$ social-related variables such as criminality, ${ }^{7}$ and well-being and quality of life. ${ }^{26}$ Treatment participation (retention in treatment) improves outcomes of OMT, ${ }^{27}{ }^{28}$ while cycling in and out of OMT seems to be a common trajectory for many people with OUD. ${ }^{29}$

However, OMT is not a static and well-defined treatment modality. The current evidence base consists of a wide variation in outcome measures of treatment of people with OUD, across time and settings, which reduces comparability of results. ${ }^{20}$ Therefore, OMT must be evaluated over the same time period as well as in different national health system and clinical contexts, in order to consistently provide high-quality care and improve our understanding of the dynamics of OMT and outcomes. Especially, in countries from the former Eastern European region, data regarding the efficacy, tolerability and predictors of outcome for OMT remain scarce. In addition, evidence based on comparisons of the same outcomes in several risk populations and across national treatment systems is lacking. For example, an increased risk in overdose death during the induction/ cessation of treatment was observed, ${ }^{20}$ indicating that patients are especially vulnerable during periods of treatment transition, while others have observed very low rates of mortality during the first period after treatment start and treatment termination. Therefore, use of data from multiple nationwide health and population registers is an important new approach with great potential to further improve the knowledge base. ${ }^{30-32}$ Further, use of multiple national data prevents potential confounding and classification bias in comparison with mortality risk between OMT, including periods of cycling in and out of treatment. ${ }^{25} 28$ Finally, linking data from multiple registers allows for large unselected study populations, and for identification of relevant comparison groups and comparable outcome indicators.

The aim of this project is to identify factors associated with important beneficial outcomes for OMT patients across settings, in order to further improve treatment practices and our understanding of the key elements of treatment success.

\section{Project objectives}

The main objective is to study OMT adherence trajectories, comorbidity and mortality, based on large patient cohorts in the Czech Republic, Norway and Denmark.

Specifically, in the national OMT cohorts, we plan to:

1. Describe OMT, OMT patients, OMT adherence trajectories and predictors of these trajectories.

2. Study comorbidity in OMT patients while observing other somatic and mental morbidities.
3. Investigate crude mortality rate for all cause and causespecific mortality.

Different OMT adherence trajectories will be characterised by OMT entry, drop-out, re-entry, time in OMT and OMT termination.

All three objectives will involve stratified analysis with regard to treatment with methadone, buprenorphine and buprenorphine in combination with naloxone, or switching between the different OMT medications.

\section{METHODS AND ANALYSIS \\ Project period}

Data cleaning and the first analyses are planned for the period between February and May 2021. The planned end date of the project is December 2024. The project period may be extended with additional funding.

\section{Treatment settings}

Czech Republic

OMT was introduced in one treatment facility in the Czech Republic in 1997 and became a national standard treatment modality in 2000. In prison, OMT was piloted in 2006 and has been used regularly since $2008 .^{33}$ Two OMT medications, methadone and buprenorphine, both administered orally, are used, and these are represented by six products on the current market. Methadone is available to patients through specialised OMT clinics with varying levels of threshold, but is in general accompanied by psychosocial services. Medication that contains buprenorphine can be prescribed by the physician, and its use is more widespread but with less formal follow-up services. However, the availability and affordability of OMT is limited, since methadone is used only in 12 specialised centres in the country, and buprenorphine is subject to a strict health insurance scheme, so that a majority of the patients in reality have to pay full price for their medication. ${ }^{34}$ Provision of OMT is monitored and governed by the Standard of Substitution Treatment issued by the Ministry of Health in cooperation with Society for Addiction Treatment of the Czech Medical Association.

\section{Norway}

OMT was implemented as a national programme in 1998. ${ }^{35}$ Treatment is initiated at the specialist (secondary care) level. In most cases, after a period of stabilisation, the patient is transferred to the primary care level with regular follow-up and prescriptions by the general practitioner and the social services in the municipality. The Norwegian OMT programme has gradually become more low-threshold and harm reduction oriented, with very few patients discharged from treatment, regardless of ongoing drug use during enrolment. ${ }^{35}$ There is no time limit on OMT in Norway, and patients may be provided with treatment in a lifelong perspective. The Norwegian OMT model has a clear 'rehabilitative' goal that includes the provision of psychosocial services, and aims that patients improve their health and well-being to best reach their 
individual potential. Since 2010, the Norwegian OMT has had a national OMT guideline, issued by the Directorate of Health (scheduled for an update in 2021). OMT is free and publicly funded for all patients in Norway.

\section{Denmark}

OMT was introduced as a treatment option for patients with OUD in Denmark in $1970 .{ }^{36}$ At first, methadone was the only available OMT medication, but since 2008, buprenorphine has also been available. ${ }^{37}$ In addition to buprenorphine and methadone, heroin-assisted treatment has been available in Denmark since 2009. ${ }^{38}$ As part of the Danish welfare services, OMT is free and publicly funded, and the Danish law states that the municipalities must initiate treatment and formulate a treatment plan for OUD within 2 weeks of the first treatment request. 390 Although two different sets of policies govern OMT in Denmark-health regulations and social service regulations-practitioners consider OMT as an integrated treatment that includes both healthcare and psychosocial services. The aim of OMT is to support the patients in having a meaningful and satisfying life in a way that is defined and controlled by the patient themselves. ${ }^{40}$ This may also include a lifelong involvement in OMT.

\section{Design}

All research questions will be answered using a national cohort approach, linking the registers' data sources by using the unique personal identification number (the so-called birth number) assigned to all residents at birth in the Czech Republic, Norway and Denmark.

\section{Data sources}

The overview with description of relevant national registers in the Czech Republic, Norway and Denmark is presented in table 1 . In all countries, physicians and pharmacies are obliged by law to report data to the nationwide registers. Data are collected prospectively and without informed consent. Earlier studies indicate that the quality of data in the registers from Norway and Denmark is moderate to high, depending on the condition or treatment studied. ${ }^{41-46}$

\section{Study population and size}

The Czech Republic cohort

Using data from the entire Czech Republic, the OMT population will be obtained from the National Register of Addiction Treatment (table 1). Approximately 4000 men and 1700 women enrolled in OMT in the period 20002020 will be included in the study. More than half of the patients were between 30 and 39 years.

\section{The Norwegian OMT cohort}

In Norway, the OMT population will be defined by the Norwegian Prescription Database (table 1). By 2020, there was around 7900 patients enrolled in treatment, of which $30 \%$ were women, with a mean age of 46 years.

\section{The Danish registry cohort}

The OMT population will be defined by the Danish Registry of Drug Abusers Undergoing Treatment (table 1). By 2018 (latest data), 6656 patients had been enrolled in OMT. During 2018, 737 patients enrolled in OMT, of which $21 \%$ were women, with a mean age of 46 years. ${ }^{47}$

\section{Comparison groups}

Regarding objectives 2 and 3, patients with OUD (International Classification of Diseases, 10th Revision (ICD10), F11 code-opioids-related disorders) not receiving OMT, patients with other drug use disorders (ICD-10, F10-F19 codes-mental and behavioural disorders due to psychoactive substance use, except F11), and the general drug non-using populations will be used as comparison groups. In some analyses, the different OMT adherence trajectories will be compared with each other.

\section{Patient and public involvement}

The study team members are in contact with patients and public representatives regarding design, running the study and interpretation of the study findings. Moreover, the patient representatives will be encouraged to disseminate the findings within their patient networks.

\section{Outcome variables}

The following outcome variables will be obtained from the respective registers (see table 1 ).

\section{OMT adherence trajectories}

Characterised by OMT entry, drop-out, re-entry, time in OMT and OMT termination.

\section{Comorbidity}

Somatic and psychiatric comorbidity (eg, infections, immune disorders, cardiovascular and respiratory diseases, epilepsy and neoplasms, neurological disorders, anxiety, depression, sleep problems) (based on ICD-10 codes (secondary care), International Classification of Primary Care, Second Edition codes (primary care), and prescribed/dispensed drugs as proxy of disease).

\section{Mortality}

All-cause mortality and specific morality (eg, overdose deaths, accidents, cardiovascular disease/cancer, others) (based on ICD-10 codes).

\section{Analysis strategy}

All data will be analysed using an individual participant data meta-analyses method. ${ }^{48}$ Methodologically, this approach allows for applying consistent inclusion/exclusion criteria applied across countries, an approach that has successfully been applied in other studies. ${ }^{48-50}$ This method has several advantages, among others: (a) as data are analysed locally in the first step, it overcomes the privacy hurdles associated with sharing individual-level data across countries and jurisdictions; (b) involvement of local researchers provides an opportunity to convey the 
Table 1 Potential data sources used in the OMT registry-based study in the Czech Republic, Norway and Denmark

\begin{tabular}{|c|c|c|c|}
\hline & Czech Republic & Norway & Denmark \\
\hline \multirow{2}{*}{$\begin{array}{l}\text { Information about } \\
\text { OMT user and OMT } \\
\text { drug }\end{array}$} & $\begin{array}{l}\text { National Register of Addiction } \\
\text { Treatment }\end{array}$ & The Norwegian Prescription Database (NorPD) & $\begin{array}{l}\text { The Danish Registry of Drug Abusers Undergoing } \\
\text { Treatment }\end{array}$ \\
\hline & $\begin{array}{l}\text { Information about patients who } \\
\text { receive OMT, date of treatment } \\
\text { initiation and termination and } \\
\text { type of OMT drug }\end{array}$ & $\begin{array}{l}\text { Prescription drugs dispensed at pharmacies, } \\
\text { all opioids dispensed to outpatients are } \\
\text { registered. OMT opioids can be identified by } \\
\text { use of ATC codes and Nordic article number. } \\
\text { It is estimated that about } 90 \% \text { of the OMT } \\
\text { patients are identified using the NorPD. } \\
\text { Use of prescription drugs as proxy of disease } \\
\text { is possible in two different situations. For } \\
\text { reimbursed drugs, the ICD- } 10 \text { or ICPC-2 } \\
\text { diagnosis code of the disorder that is treated } \\
\text { has to be written on the prescription. The } \\
\text { other option is to use drugs that only have } \\
\text { one indication like for instance insulin for } \\
\text { treating diabetes. }\end{array}$ & $\begin{array}{l}\text { Information on patients who are enrolled in outpatient } \\
\text { treatment for drug use disorders, including information } \\
\text { about patients who receive OMT, date of treatment } \\
\text { initiation/termination and type of OMT drug }\end{array}$ \\
\hline $\begin{array}{l}\text { Information about } \\
\text { comorbidity }\end{array}$ & $\begin{array}{l}\text { National Register of Inpatient } \\
\text { Treatment }\end{array}$ & $\begin{array}{l}\text { National Primary Care Reimbursement } \\
\text { System }\end{array}$ & The Danish National Prescription Register \\
\hline
\end{tabular}
comorbidity Treatment

Information on every episode of all types of hospitalisations, including information on dates of admission and discharge from hospital. All diagnoses (primary and secondary) and procedures (ICD-10).

Information System Infections (EPIDAT)

Information on all notifications of selected serious infections verified cases, suspected diseases, carrier of an infection and deaths.

Diagnosis (ICPC-2) from claims from general practitioners and other health service

Information on all individual prescription drugs dispensed through pharmacies to patients outside of institutions providers, such as emergency rooms, date of such as hospitals and drug treatment facilities consultation

The National Patient Register

Information on all patients receiving hospitallevel care in both inpatient and outpatient facilities, and acute and emergency services for mental and somatic illnesses. Primary and secondary diagnoses (ICD-10), date of admission, date of discharge
The Danish National Patient Register

Information on all patients receiving hospital-level care in both inpatient and outpatient facilities, and acute and emergency services for somatic illnesses. Primary and secondary diagnoses (ICD-10), date of admission, date of discharge

National Register of Reimbursed

Health Services

Also includes information on prescribed drugs reimbursed within the health insurance system.

$\begin{array}{ll}\begin{array}{ll}\text { Information about } \\ \text { death and cause of } \\ \text { death }\end{array} & \begin{array}{l}\text { National General Mortality } \\ \text { Register } \\ \text { Cause of death diagnosis (ICD- } \\ \text { 10), date of death }\end{array} \\ \begin{array}{l}\text { Information about } \\ \text { socioeconomic } \\ \text { status }\end{array} & \begin{array}{l}\text { Czech Statistical Office } \\ \text { Demographic data, country of } \\ \text { birth, education, work situation, } \\ \text { income, disability pension }\end{array}\end{array}$

Cause of Death Registry

Cause of death diagnosis (ICD-10), date of death

\section{Statistic Norway}

Demographic data, country of birth, education, work situation, income, disability pension

ATC, Anatomical Therapeutic Chemical; ICD-10, International Classification of Diseases, 10th Revision; ICPC-2, International Classification of Primary Care, Second Edition; OMT, opioid maintenance treatment.

nuances of local and national data; (c) allows for consistent and realistic adjustment for confounding factors that may explain differences in findings across countries; and (d) allows for increased clinical relevance of findings by providing the opportunity to explore clinical questions across different national contexts and times. ${ }^{48} 50$
Objective 1: OMT adherence trajectories

First, we will identify all OMT patients in each country. Following this, we will describe the national OMT cohorts in general with regard to pharmacological treatment provided and background characteristics of the patients. In addition, we will identify different OMT adherence 
trajectories based on entry to treatment, drop-out, re-entry, total time in OMT and OMT termination. We will study associations between health, demographic and socioeconomic factors, different OMT adherence trajectories, and psychiatric and somatic comorbidity.

\section{Objective 2: comorbidity}

We will investigate comorbidity and use of healthcare services in the different national OMT cohorts by studying the number of contacts with healthcare services for the OMT groups and the comparison groups. Also, we will study somatic and psychiatric comorbidity by studying different specific diagnoses and compare them with comorbidity among the comparison groups.

\section{Objective 3: mortality and cause of death}

We will compare mortality and causes of death in the national OMT cohorts. Patients in OMT will be compared with patients who have been in OMT, but have dropped out of or terminated treatment. Mortality will also be compared with mortality among patients with drug use disorders not in OMT and with the general population.

Regarding all three objectives, harmonised and firmlevel data will be pooled across countries in order to investigate between-country variations in a fully consistent manner but with respect to privacy and confidentiality restrictions on micro-data.

\section{Statistical analyses}

\section{Descriptive analysis}

Statistical analyses will include descriptive analyses of the pharmacological OMT treatment and the characteristics of the study populations in the different national cohorts.

\section{OMT adherence trajectories}

First, we will do explorative examinations of OMT with respect to initiation, time in treatment, number of dropouts and re-entries, and terminations in the different national cohorts. Based on these results, we will assign patients to different OMT adherence trajectory groups. Potential factors associated with different treatment trajectories groups will be examined using multinomial logistic regression analysis. The coefficients will be interpreted in terms of relative risk ratios with $95 \%$ CIs. We will run univariate models for all covariates and one multivariate model. In the multivariate model, age, gender, psychiatric and somatic comorbidity, and use of other prescription drugs with abuse potential will be adjusted for.

\section{Comorbidity}

We will study comorbidity in a defined period before OMT initiation, during treatment and after termination of treatment. Comorbidity will be measured as number of contacts and diagnosis from primary (Czech Republic and Norway) and secondary healthcare. The number of contacts and proportions with selected groups of diagnosis will be compared by t-test or $\mathrm{X}^{2}$ test. For selected diagnosis, we will also study the proportion (with 95\% CI) receiving adequate prescription drug treatment. We will compare comorbidity in OMT patients in the different national cohorts. All analyses will be stratified by gender and age group.

\section{Mortality}

Patients initiating OMT often cycled in and out of treatment. We will estimate the crude mortality rates (CMRs) and $95 \%$ CIs as number of deaths per 1000 person-years in-treatment and post-treatment. ${ }^{51}$ We will define the following time at risk periods: in-treatment: the period between treatment start and death, from treatment start to termination of treatment, or from treatment start and the end of the observation period. As the same patient may contribute to multiple observation periods, all treatment periods are included in the analysis. Post-treatment: the period between termination of treatment and death, from termination of treatment to another treatment start, or from termination of treatment to the end of the observation period. The same patient can contribute to several out-of-treatment periods between treatment episodes, and all these between-periods are included in the analysis. In order to compare CMRs between in-treatment and post-treatment, crude rate ratios will be calculated by dividing one mortality rate by another.

We will also compare CMRs between different subgroups, for example, women and men and age groups, and between patient groups receiving methadone, buprenorphine, and buprenorphine and naloxone patients. To compare mortality between OMT patients and the general population, standardised mortality ratio will be calculated.

Statistical analyses will be performed on SPSS (V.24) and Stata packages (V.14.2). Analysis and publication of results will follow the Strengthening the Reporting of Observational Studies in Epidemiology guidelines. ${ }^{52}$

\section{Ethics and personal data protection}

Approval from the respective ethics committees in: the Czech Republic (36/19GrantAZVVES20201.LFUK) and Norway (REK number 2019/656/REK sør-øst C) has already been obtained. In Denmark, ethical evaluation is not required by law, but was approved by the national data authority (journal number: 2013540288, updated 13 March 2020). All disseminations from the project will be on group-based information, and no individual participants will be recognisable.

All relevant national registers include independent and prospectively collected information on the health and socioeconomic status of all inhabitants in the Czech Republic, Norway and Denmark. Data of our interest are already available in the national registers. Data in the project will be used in pseudo-anonymised form. Each country will manage its own country-specific data. Applicable to all, data will be directly loaded to and saved on an information technology platform specifically dedicated to secure storage of sensitive research data at the respective institutions. 


\section{Data management}

Original data received from the health registers' data administrators will be checked for consistency, and series of automatic range checks will be performed until the database is considered clean; all data procedures in this phase will be tracked. A data backup will be performed periodically during the study. Only designated investigators will be allowed access to data.

\section{Strengths and limitations}

Nationwide registers provide a great potential to study large cohorts and examine longitudinal outcomes with less selection bias and reduced risk of recall bias compared with clinical studies. At a global level, only some countries have nationwide registers that cover both health data and socioeconomic status. The Scandinavian countries and the Czech Republic have such registers. Registry-based cohort studies enable us to control for important confounding factors, such as socioeconomic and health status. The Czech and Scandinavian registers are comparable and provide great opportunities to conduct innovative research, for instance, comparing OMT patients with similar characteristics across national cohorts. Further, the Czech and Scandinavian registers provide a wealth of comparable data regarding relevant ICD-10 health conditions that allows us to study comorbidity and mortality in OMT populations on a national level and across countries, and to compare data with the general population and other subpopulations. In addition, the current evidence regarding OMT is insufficient in the countries representing the former Eastern European region. Last but not least, the study will allow us to examine and compare different treatment traditions in OMT, for example, the more liberal OMT in Denmark, ${ }^{53}$ the historically restrictive OMT, however, with gradual transition into a more liberal since 2010 in Norway, ${ }^{20}$ and the less available and affordable OMT in the Czech Republic. $^{34}$

Larger patient cohorts increase the statistical power, and the analyses of combined multiple data sources increase the richness of information available and enable to control for important factors across countries, such as culture-specific factors and differences in country-specific healthcare systems. Furthermore, an international registry-based study design increases the generalisability of the findings.

There are, however, some drawbacks of registry cohort studies. The main limitations are that important information can be under-reported, reported in insufficient format or be lacking in the national health registers (eg, use of licit and illicit drugs). Furthermore, there may be potential limitations in controlling for all sources of bias and unmeasured confounders (eg, lifestyle factors that may potentially affect the outcomes in people with OUD).$^{54}$

We define OMT patients in a different way in the Norwegian data than in the Czech Republic and Denmark. By using prescription data for OMT medication, uncertainty of the date of initiation and termination of treatment may arise. However, preliminary analyses show that OMT patients are mainly dispensed OMT medication quite frequently (weekly or every second week). So we assume that as long as the patients are being prescribed OMT medication, they are enrolled in OMT.

Norway and Denmark have prescription databases that have been used for research purposes for years. In the Czech Republic, data on prescription drugs for the past 10 years were made available in 2020 , and there is limited experience in using this data in research. This project will be the first to use such data.

\section{Implications for interventions and future policy}

Physicians and other healthcare providers within the healthcare system need updated and latest evidence-based knowledge regarding the models of care and benefits of OMT and treatment of OUD in general. The project will provide invaluable information, not only in the respective countries, but internationally.

The research outcomes will improve the quality of OMT for people with OUD and contribute to the reduction of health burden of disease and mortality, improved quality of life and reduce social exclusion of people with OUD. It is also important to identify risk factors associated with treatment discontinuation, relapse to drug use, causes of premature death, and psychiatric and somatic disorders. Research in this field has the potential to identify significant areas of benefit, to break the relapsing nature of OUD and, thus, reduce public health burden among people with OUD.

The project has great potential for improving the utilisation of the national registers by providing feedback on the quality and validity of the collected data. The project is unique in its utilisation of the number of nationwide registers in a database-linkage project, and will be the first in the history of the health information system in the Czech Republic and European Union-except Scandinavian countries.

Comorbidity should be generally accepted as a complex multifactorial issue, and its individual components cannot be completely separated in any research. Similarly, the treatment models vary between countries and within countries, both by region and over time. Despite the complexity, it is well known that OMT (using well-tested opioid agonists of defined quality, dosage, release form, effects) reduces the harm related to OUD in comparison with ongoing use of illicit drugs outside of treatment. ${ }^{55}$

In conclusion, we present a comparative international project initiative to study OMT in the Czech Republic, Norway and Denmark. Investigations will improve our knowledge of how national treatment systems evolve over time regarding patient flow and OMT participation and outcomes. By identifying profiles and time phases of models of OMT and its outcomes, we can better guide clinicians and inform decision-makers and policymakers. 
Contributors RG, SS and MHa designed the project. All other authors, VM, BN, CT, $\mathrm{BT}, \mathrm{MHe}, \mathrm{AB}, \mathrm{TC}, \mathrm{JM}$ and JJ, contributed to the specific design of the project. RG, SS and $\mathrm{MHa}$ wrote the study protocol with substantial critical input from BN, CT, BT, AB and TC. The remaining authors, VM, MHe, JM and JJ, contributed to refinement of the paper. Description of data sources, including table 1, and the study population and size was drafted by SS and completed by BN, JJ, MHa and CT. The Statistical analyses section was drafted by SS and refined by BN, AB, TC and BT. Review of literature was conducted by RG, MHa, JM, AB and MHe. All authors read and approved the final version of the manuscript.

Funding The project was supported by the Ministry of Health of the Czech Republic (grant no. NU20-09-00066); the institutional support programme (Progress no. Q06/LF1); and the Specific University Research programme (grant no. 260500).

\section{Competing interests None declared.}

Patient and public involvement Patients and/or the public were not involved in the design, or conduct, or reporting, or dissemination plans of this research.

Patient consent for publication Not required.

Provenance and peer review Not commissioned; externally peer reviewed.

Open access This is an open access article distributed in accordance with the Creative Commons Attribution Non Commercial (CC BY-NC 4.0) license, which permits others to distribute, remix, adapt, build upon this work non-commercially, and license their derivative works on different terms, provided the original work is properly cited, appropriate credit is given, any changes made indicated, and the use is non-commercial. See: http://creativecommons.org/licenses/by-nc/4.0/.

\section{ORCID iD}

Roman Gabrhelík http://orcid.org/0000-0002-7672-3753

\section{REFERENCES}

1 Degenhardt L, Grebely J, Stone J, et al. Global patterns of opioid use and dependence: harms to populations, interventions, and future action. Lancet 2019;394:1560-79.

2 James SL, Abate D, Abate $\mathrm{KH}$, et al. Global, regional, and national incidence, prevalence, and years lived with disability for 354 diseases and injuries for 195 countries and territories, 1990-2017: a systematic analysis for the global burden of disease study 2017 . Lancet 2018;392:1789-858.

3 Vereinte Nationen. Büro für Drogenkontrolle und Verbrechensbekämpfung. World drug report 2018, 2018.

4 Zibbell JE, Asher AK, Patel RC, et al. Increases in acute hepatitis C virus infection related to a growing opioid epidemic and associated injection drug use, United States, 2004 to 2014. Am J Public Health 2018;108:175-81.

5 Skeie I, Brekke M, Lindbaek M, et al. Somatic health among heroin addicts before and during opioid maintenance treatment: a retrospective cohort study. BMC Public Health 2008;8:43.

6 Higgins $\mathrm{C}$, Smith $\mathrm{BH}$, Matthews K. Comparison of psychiatric comorbidity in treatment-seeking, opioid-dependent patients with versus without chronic pain. Addiction 2020;115:249-58.

7 Bukten A, Skurtveit S, Gossop M, et al. Engagement with opioid maintenance treatment and reductions in crime: a longitudinal national cohort study: opioid maintenance treatment and crime. Addiction 2012;107:393-9.

8 Skjaervø I, Skurtveit S, Clausen T, et al. Substance use pattern, selfcontrol and social network are associated with crime in a substanceusing population. Drug Alcohol Rev 2017;36:245-52.

9 Nutt DJ, King LA, Phillips LD, et al. Drug harms in the UK: a multicriteria decision analysis. Lancet 2010;376:1558-65.

10 Taylor M, Mackay K, Murphy J, et al. Quantifying the RR of harm to self and others from substance misuse: results from a survey of clinical experts across Scotland. BMJ Open 2012;2:e000774.

11 van Amsterdam J, Opperhuizen A, Koeter M, et al. Ranking the harm of alcohol, tobacco and illicit drugs for the individual and the population. Eur Addict Res 2010;16:202-7.

12 Degenhardt L, Bucello C, Mathers B, et al. Mortality among regular or dependent users of heroin and other opioids: a systematic review and meta-analysis of cohort studies. Addiction 2011;106:32-51.

13 Degenhardt L, Larney S, Randall D. Causes of death in a cohort treated for opioid dependence between 1985 and 2005: mortality among opioid-dependent people. Addiction 2014;109:90-9.

14 World Health Organization. International narcotics control board, United nations office on drugs and crime, editors. guidelines for the psychosocially assisted pharmacological treatment of opioid dependence. Geneva: World Health Organization, 2009: 111.
15 European Monitoring Centre for Drugs and Drug Addiction. Health and social responses to drug problems: a European guide. [online]. LU: Publications Office, 2017. https://data.europa.eu/doi/

16 European Monitoring Centre for Drugs and Drug Addiction. European drug report 2019: trends and developments [online], 2019. Available: https://data.europa.eu/doi/ [Accessed 17 Aug 2019].

17 Skurtveit S, Nechanská B, Handal M, et al. Hospitalization of children after prenatal exposure to opioid maintenance therapy during pregnancy: a national registry study from the Czech Republic. Addiction 2019;114:1225-1235 https://onlinelibrary.wiley.com/doi/ abs/

18 Nechanská B, Mravcík V, Skurtveit S, et al. Neonatal outcomes after fetal exposure to methadone and buprenorphine: national registry studies from the Czech Republic and Norway. Addiction 2018;113:1286-94.

19 Jones HE, Kaltenbach K, Heil SH, et al. Neonatal abstinence syndrome after methadone or buprenorphine exposure. $N$ Engl J Med 2010;363:2320-31.

20 Bukten A, Stavseth MR, Clasuen T. From restrictive to more liberal: variations in mortality among patients in opioid maintenance treatment over a 12-year period. BMC Health Serv Res 2019;19 https://bmchealthservres.biomedcentral.com/articles/

21 Clausen T, Anchersen K, Waal H. Mortality prior to, during and after opioid maintenance treatment (OMT): a national prospective crossregistry study. Drug Alcohol Depend 2008;94:151-7.

22 Connock M, Juarez-Garcia A, Jowett S, et al. Methadone and buprenorphine for the management of opioid dependence: a systematic review and economic evaluation. Health Technol Assess 2007;11 https://www.journalslibrary.nihr.ac.uk/hta/hta11090/

23 Degenhardt L, Randall D, Hall W, et al. Mortality among clients of a state-wide opioid pharmacotherapy program over 20 years: risk factors and lives saved. Drug Alcohol Depend 2009;105:9-15.

24 Marotta PL, McCullagh CA. A cross-national analysis of the association between years of implementation of opioid substitution treatments and drug-related deaths in Europe from 1995 to 2013. Eur $J$ Epidemiol 2018;33:679-88.

25 Sordo L, Barrio G, Bravo MJ, et al. Mortality risk during and after opioid substitution treatment: systematic review and meta-analysis of cohort studies. BMJ 2017;357:j1550.

26 Maglione MA, Raaen L, Chen C, et al. Effects of medication assisted treatment (MAT) for opioid use disorder on functional outcomes: a systematic review. J Subst Abuse Treat 2018;89:28-51.

27 Bukten A, Skurtveit S, Waal H, et al. Factors associated with dropout among patients in opioid maintenance treatment (OMT) and predictors of re-entry. A national registry-based study. Addict Behav 2014;39:1504-9.

28 Busch M, Klein C, Uhl A, et al. Retention in the Austrian opioid agonist treatment system: a national prospective cohort study. Harm Reduct J 2021:18:25.

29 Nordt C, Wiessing L, Kuijpers W, et al. Long-term opioid agonist treatment participation after first treatment entry is similar across 4 European regions but lower in Non-Nationals. Eur Addict Res 2018;24:173-83.

30 Gabrhelík R, Nechanská B, Mravcík V, et al. A unique opportunity to study short- and long-term consequences in children prenatally exposed to illicit drugs and opioid maintenance treatment using Czech and Scandinavian registers. Cent Eur J Public Health 2016;24:248-51.

31 McMahon AW, Dal Pan G. Assessing drug safety in children - the role of real-world data. N Engl J Med 2018;378:2155-7.

32 Mahic M, Hernandez-Diaz S, Wood M, et al. In utero opioid exposure and risk of infections in childhood: a multinational Nordic cohort study. Pharmacoepidemiol Drug Saf 2020;29:1596-604.

33 Mravcik V, Orlíková B. Substituční Léčba Závislosti Na Opioidech V ČR: Kritický Pohled. Čs a slov Psychiat 2019;115:53-8.

34 Mravcík V, Janíková B, Drbohlavová B, et al. The complex relation between access to opioid agonist therapy and diversion of opioid medications: a case example of large-scale misuse of buprenorphine in the Czech Republic. Harm Reduct J 2018;15:60.

35 Waal $\mathrm{H}$. Merits and problems in high-threshold methadone maintenance treatment. Eur Addict Res 2007;13:66-73.

36 European Monitoring Centre for Drugs and Drug Addiction. European drug report 2020: trends and developments [online]. LU: Publications Office, 2020. https://data.europa.eu/doi/10.2810/420678

37 European Monitoring Centre for Drugs and Drug Addiction. Denmark country overview, 2016. Available: https://www.emcdda.europa.eu/ node/2580_ro\#treatmentResponses [Accessed 20 Oct 2020]

38 Sundhedsstyrelsen. Evaluering AF ordning Med lægeordineret heroin TIL stofmisbrugere 2010-2012 2013.

39 Skretting A, Rosenqvist P. Shifting focus in substitution treatment in the Nordic countries. Nord Stud Alcohol Drugs 2010;27:581-98. 
40 Sundhedsstyrelsen. Vejledning TIL læger, Der behandler opioidafhængige patienter Med substitutionsmedicin: lovkrav OG anbefalinger. Sundhedsstyre/sen 2017.

41 Furu K, Karlstad Øystein, Skurtveit S, et al. High validity of motherreported use of antiasthmatics among children: a comparison with a population-based prescription database. J Clin Epidemiol 2011;64:878-84.

42 Furu K, Wettermark BjÃ $\mid r n$, Andersen M, et al. The Nordic countries as a cohort for Pharmacoepidemiological research. Basic Clin Pharmacol Toxicol 2010;106:86-94.

43 Hallas J. Conducting pharmacoepidemiologic research in Denmark. Pharmacoepidemiol Drug Saf 2001;10:619.

44 Schmidt M, Pedersen L, Sørensen HT. The Danish civil registration system as a tool in epidemiology. Eur J Epidemiol 2014;29:541-9.

45 Schmidt M, Schmidt SAJ, Sandegaard JL, et al. The Danish national patient registry: a review of content, data quality, and research potential. Clin Epidemiol 2015;7:449.

46 Pedersen AG, Ellingsen CL. Datakvaliteten i Dødsårsaksregisteret. Tidsskriftet 2015;135:768-70.

47 Sundhedsstyrelsen. Stofmisbrugsbehandling - efterspørgsel og tilgængelighed. Narkotikasituationen i Danmark - delrapport 3 [online]. Sundhedsstyrelsen, 2020. https://www.sst.dk/-/media/ Udgivelser/2020/Narkotikasituationen/Narkorapporten-03.ashx?la= da\&hash=1E075F4218C3B4CA2E3B38A28D9B56B6D2BB428C

48 Borschmann R, Tibble H, Spittal MJ, et al. The mortality after release from incarceration Consortium (MARIC): protocol for a multi-national, individual participant data meta-analysis. Int J Popul Data Sci 2020;5:1145.

49 Kivimäki M, Nyberg ST, Batty GD, et al. Job strain as a risk factor for coronary heart disease: a collaborative meta-analysis of individual participant data. Lancet 2012;380:1491-7.

50 Riley RD, Lambert PC, Abo-Zaid G. Meta-analysis of individual participant data: rationale, conduct, and reporting. BMJ 2010;340:c221.

51 Rothman KJ, Greenland S, Lash TL. Modern epidemiology. 3rd edn. Philadelphia, Baltimore, New York: Wolters Kluwer Health, Lippincott Williams \& Wilkins, 2008: 758.

52 von Elm E, Altman DG, Egger M, et al. The strengthening the reporting of observational studies in epidemiology (STROBE) statement: guidelines for reporting observational studies. Int J Surg 2014;12:1495-9.

53 Tjagvad C, Skurtveit S, Linnet K, et al. Methadone-related overdose deaths in a liberal opioid maintenance treatment programme. Eur Addict Res 2016;22:249-58.

54 Mravcík V, Nechanská B, Gabrhelík R, et al. Socioeconomic characteristics of women with substance use disorder during pregnancy and neonatal outcomes in their newborns: a nationa registry study from the Czech Republic. Drug Alcohol Depend 2020;209:107933.

55 Kivimies K, Repo-Tiihonen E, Kautiainen $\mathrm{H}$, et al. Comorbid opioid use is undertreated among forensic patients with schizophrenia. Subst Abuse Treat Prev Policy 2018;13:39. 